\title{
Optoacoustic imaging of Glucagon-like Peptide 1 Receptor with a near-infrared exendin-4 analog
}

Running title: Optoacoustic imaging of GLP-1R

Sheryl Roberts ${ }^{1}$, Eshita Khera ${ }^{2}$, Crystal Choi ${ }^{1}$, Tejas Navaratna ${ }^{2}$, Jan Grimm ${ }^{1,3,4,5}$, Greg M. Thurber ${ }^{2,6}$, Thomas Reiner ${ }^{1,4,7 *}$

${ }^{1}$ Department of Radiology, Memorial Sloan Kettering Cancer Center, New York, NY, 10065, USA

${ }^{2}$ Department of Chemical Engineering, University of Michigan, Ann Arbor, Michigan, United States

${ }^{3}$ Department of Molecular Pharmacology, Memorial Sloan Kettering Cancer Center, New York, New York, USA

${ }^{4}$ Department of Radiology, Weill Cornell Medical College, 1300 York Avenue, New York, New York.

${ }^{5}$ Pharmacology Program, Weill Cornell Medical College, 1300 York Avenue, New York, New York, 10065, USA

${ }^{6}$ Department of Biomedical Engineering, University of Michigan, Ann Arbor, Michigan, Unites States

${ }^{7}$ Chemical Biology Program, Memorial Sloan Kettering Cancer Center, New York, New York 10065

Correspondence should be addressed to:

*Thomas Reiner

1275 York Avenue

New York, NY, 10065

reinert@mskcc.org; (P) 1-646-888-3461; (F) 6464420408 


\section{ABSTRACT}

Limitations in current imaging tools have long challenged the imaging of small pancreatic islets in animal models. Here, we report the first development and in vivo validation testing of a broad spectrum and high absorbance near infrared optoacoustic contrast agent, E4x12-Cy7. Our near infrared tracer $(E 4 \times 12-C y 7)$ is based on the amino acid sequence of exendin-4 and targets the glucagon-like peptide-1 receptor (GLP-1R). Cell assays confirmed that $\mathrm{E} 4 \times 12-\mathrm{Cy} 7$ has a high binding affinity $\left(\mathrm{IC}_{50}=4.6 \pm 0.8 \mathrm{nM}\right)$. Using the multispectral optoacoustic tomography (MSOT), we imaged E4×12-Cy7 and optoacoustically visualized ß-cell insulinoma xenografts in vivo for the first time. In the future, similar optoacoustic tracers that are specific for ß-cells and combines optoacoustic and fluorescence imaging modalities could prove to be important tools for monitoring the pancreas for the progression of diabetes.

\section{Keywords}

optoacoustic | diabetes | ß-cell imaging | near infrared | exendin-4 | sonophore | MSOT 


\section{INTRODUCTION}

Imaging does not presently play a prominent role in diabetes diagnostics. The current diagnosis for type 1 or 2 diabetes and prediabetes is based on the patient's medical history and blood screening in the form of an AC1 test(1). However, the prevalence of diabetes is expected to drive the demand and use of molecular imaging in order to facilitate the diagnosis and monitoring of the disease. Type 1/2 diabetes for all age-groups worldwide is projected to rise from 171 million in 2000 to 366 million in 2030(2). The rise in the incident of diabetes- a major cause of blindness, kidney failure, heart attacks, stroke and lower limb amputation(3)- has not been met with a comparable, parallel refinement in diagnostic tools for its early detection. Unlike computed tomography (CT), magnetic resonance imaging $(\mathrm{MRI})$, ultrasonography and intravital microscopy $(4,5)$, which have played major roles, in the field of diabetes imaging, optoacoustic imaging is thus far underrepresented among diagnostic tools. Optoacoustic glucose sensing techniques in blood and plasma samples, much like the AC1 test, have been reported as early as in 1999(6). Few studies, however, focus on newly developed techniques of optoacoustic spectrometry and specific spectral unmixing algorithms intended to sense glucose(7-9). While glucose sensing remains the most effective method for the detection of diabetes $(10)$, the direct visualization of $ß$-cells remains a high priority, as it promises to diagnose occult diabetes before metabolic imbalance and dysregulated blood levels manifest.

Beta-cells are key regulators of glucose balance via secretion of insulin, and ß-cell dysfunction leads to chronic diseases, type 1 and type 2 diabetes(11,12). Prolonged high 
blood sugar levels can damage major organs, moreover and lead to the development of diseases and complications beyond diabetes $(13,14)$. Although, molecular imaging of $ß-$ cell function has always garnered interest, accurate in vivo ß-cell mass (BCM) identification and quantification via molecular imaging remains elusive for three main reasons: (1) the tracer of choice must be specific for ß-cells. (2) ß-cells make up only 1$2 \%$ of the pancreas, and (3) ß-cells are located deep in the pancreas, which itself is located between major organs that are involved in clearance pathways (liver, spleen and nested between parts of the GI tract), precluding easy access for imaging. Furthermore, intravital microscopy requires invasive surgery due to light scattering, and positron emission tomography (PET) requires the use of radioactive materials. Thus far, in vivo intravital imaging and PET imaging methods have played only preclinical(15-17) and early-phase clinical roles in studying the dynamic processes of pancreatic tissue $(18,19)$.

Among other well-studied receptors(20-22), glucagon-like peptide-1 receptors (GLP-1R) is abundant on the ß-cell surface and controls for blood sugar levels through insulin secretion $(11,12)$. Since its discovery, GLP-1R has been a constant target for developing drugs. One such drug, exendin-4 (exenatide), well-known for its high affinity and efficacy is an excellent agonist (4), (23). Over the past few years, several fluorescent imaging agents based on exendin-4 have been described in the literature (24-27).

Alternatively, then, non-invasive optoacoustic tomography, which combines light and sound, could represent a viable option able to image at greater depths than other optical techniques. BCM identification needs a reliable method for non-invasive in vivo imaging 
and quantification $(28,29)$. A handful of suitable and clinically translatable two-dimensional (2D) and three-dimensional (3D) hand-held optoacoustic devices exist(30,31), including commercial options for clinical research(32-34). Based on previous approaches and motivated by the undeniable clinical need to non-invasively resolve ß-cells mass in vivo, we explored the use of exendin as a targeted vector using multi-spectral optoacoustic tomography (MSOT), a preclinical optoacoustic platform, similar to the equipment used in the clinics. Ideally, acoustic signals from the agent would overcome endogenous background signal against the inherently small populations of ß-cells. We have shown in previous studies that a number of near-infrared (NIR) dyes are suitable for optoacoustic imaging (35), (36). Such NIR scaffolds are characteristically high absorbers (typically $\varepsilon>$ $200,000 \mathrm{~cm}^{-1} \mathrm{M}^{-1}$ ) with a low quantum yield $(\theta)$ of highly packed $\pi$-conjugated aromatic systems and overlapping p-orbitals of delocalized electrons. Here, we explore the scope of NIR-exendin for applications in optoacoustic diabetes detection.

We aimed to synthesize a NIR optoacoustic sonophore, with site-specific labeling at a single position capable of targeting GLP-1R. To this end, we conjugated an exendin-4 analog to Cy7 via copper-catalyzed click chemistry, replacing the $\mathrm{K}_{12}$ position of the amino acid sequence with the azide-reactive (S)-2-amino-4-pentynoic acid $(4,26)$, to synthesize E4x12-Cy7. In order to characterize $\mathrm{E} 4 \times 12-\mathrm{Cy} 7$, we used tissue mimicking phantoms and mouse insulinoma xenografts that mimic normal expressions and function of GLP-1R akin to pancreatic ß-cells. The research described within this article incorporates our synthetic design to target GLP-1R on the surface of ß-cells using MSOT, representing the first optoacoustic visualization of insulinoma in vivo. 


\section{MATERIALS AND METHODS}

General. Liquid chromatography-mass spectrometry (LC-MS) using electrospray ionization (ESI) was recorded using a Waters instrument with SQD detector for mass identification. A lyophilizer (FreeZone 2.5 Plus, Labconco, Kansas City, MO, USA) was used for freeze drying. A BS-8000 $120 \mathrm{~V}$ high pressure (Braintree Scientific, Inc., MA, USA) multi-syringe pump was used to control the flow rate and facilitate delivery of solutions inside the phantom flow system. An automated cell counter (Beckman Coulter, Vi-Cell viability analyzer) was used for counting the number of cells. In vitro fluorescence confocal microscopy was carried out using a Leica TCS SP8. In vivo and ex vivo fluorescence imaging was carried out using a planar (2-D) IVIS Spectrum device (PerkinEImer, Waltham, MA, USA). Pymol (1.7) was used for the 3D peptide visualization. Exendin-4 structure (ID \#3C59) was taken from RCSB protein data bank (PDB).

Chemicals. All materials were obtained from Sigma Aldrich (Milwaukee, WI), unless otherwise specified. All reagents were used without further purification. Wild-type exendin-4 (H-HGEGTFTSDLSKQMEEEAVRLFIEWLKNGGPSSGAPPPS-NH ${ }_{2}$ ) was purchased as a custom peptide from Innopep (San Diego, CA). Single mutant exendin-4 (E4×12) (H-HGEGTFTSXLSKQMEEEAVRLFIEWLKNGGPSSGAPPPS-NH2), where $\mathbf{X}$ is the non-natural amino acid (S)-2-amino-4-pentynoic acid, was custom synthesized from CSBio (Menlo Park, CA). Cy7 azide (no sulfate groups) was purchased from Lumiprobe (Hallandale Beach, FL). 
Preparation of E4x12-Cy7. Preparation and characterization of Cy7 single mutant exendin (hereon referred to as $\mathrm{E} 4 \times 12-\mathrm{Cy} 7$ ) was performed using azide-alkyne click chemistry. Briefly, $300 \mathrm{nmol}$ of Cy7 azide was added to $100 \mu \mathrm{L}$ of 1:1 water/tert-butanol containing $3 \mu \mathrm{L} \mathrm{CuSO}_{4}$-THBTA (100 mM in water) and $15 \mu \mathrm{L} \mathrm{L-sodium} \mathrm{ascorbate} \mathrm{(50} \mathrm{mM}$ in water). Lastly, single mutant exendin E4x12 was added in an equal molar ratio (300 nmol) as the Cy7 azide. The mixture was reacted under magnetic stirring for 3 hours at room temperature $\left(20-25^{\circ} \mathrm{C}\right)$. Purification was performed on a Shimadzu RP-HPLC system equipped with a DGU-20A 5R degasser, SPD-20AV UV/Vis detector, a LC-6AD pump system, CTO-20A oven maintained at $30^{\circ} \mathrm{C}$, and a CBM-20A communication BUS module and using a C-18 solid phase analytical column with mobile phase of water $(0.1 \%$ trifluoroacetic acid, Buffer A) and acetonitrile (0.1\% trifluoroacetic acid, Buffer B). A 30$80 \% \mathrm{CH}_{3} \mathrm{CN}$ method (gradient: $0-18 \min 30-80 \%, 18-20 \min 80-30 \%$ ) at a flow rate of $1 \mathrm{~mL} \mathrm{~min}^{-1}$ was used to separate E4 ${ }_{12}-\mathrm{Cy} 7(\sim 16 \mathrm{~min})$ from free Cy7 azide ( $\left.21 \mathrm{~min}\right)$ and unlabeled $\mathrm{E} 4 \times 12$ ( $12 \mathrm{~min})$. Purified samples were lyophilized overnight, reconstituted in 1:1 water/acetonitrile and stored at $-20^{\circ} \mathrm{C}$ until further use. Molecular weight of the purified product was confirmed using electrospray ionization (ESI) (calculated 4786.39; found 4785.36).

Cell culture. Mouse insulinoma-derived MIN6 were used between passage 13 and 17 . Cells were grown in high-glucose DMEM containing 15\% vol/vol heat-activated FBS, 50 $\mathrm{U} / \mathrm{mL}$ penicillin, and $10 \mu \mathrm{g} / \mathrm{mL}$ streptomycin. GLP-1R positive NIT-1 mouse cells were cultured in F12K media supplemented with $10 \%(\mathrm{v} / \mathrm{v}) \mathrm{FBS}, 50 \mathrm{U} / \mathrm{mL}$ penicillin, $50 \mu \mathrm{g} / \mathrm{mL}$ streptomycin, and $1.5 \mathrm{~g} / \mathrm{L}$ sodium bicarbonate. 
In vitro binding affinity assay. Confluent NIT-1 cells were harvested using $0.05 \%$ trypsin-EDTA for 5 minutes before the enzymes were neutralized by adding FBS containing $\mathrm{F} 12 \mathrm{~K}$ media. Cells were centrifuged gently at $300 \mathrm{rpm}$ for $5 \mathrm{~min}$ at $4{ }^{\circ} \mathrm{C}$, and re-suspended in $2 \mathrm{~mL}$ PBS containing $0.5 \%$ bovine serum albumin (BSA). Cells were aliquoted and incubated in triplicate with $0.5 \%$ BSA/PBS containing E4x12-Cy 7 at varying concentrations $(0.1 \mathrm{nM}-250 \mathrm{nM})$ for 3 hours on ice. A control for non-specific binding was included by pre-incubating cells with $2 \mu \mathrm{M}$ of unlabeled wild-type exendin on ice, followed by $0.5 \%$ BSA/PBS containing $250 \mathrm{nM} \mathrm{E} 4 \times 12-\mathrm{Cy} 7$ and $2 \mu \mathrm{M}$ for 3 hours on ice. Cells were washed with $0.5 \%$ BSA/PBS and analyzed by flow cytometry (Bio-Rad ZE5 Cell Analyzer). Binding affinity ( $\left.\mathrm{IC}_{50}\right)$ was estimated using GraphPad Prism 8 using a onesite specific binding model.

Cell imaging. MIN6 $\left(1 \times 10^{5}\right.$ cells/well $)$ cells were seeded into a 96-well plate and cultured for $72 \mathrm{~h}\left(37^{\circ} \mathrm{C}\right)$. The cells were then incubated with various concentrations of $\mathrm{E} 4 \times 12-\mathrm{Cy} 7$ for 90 min at $37^{\circ} \mathrm{C}$ alone or with exendin-4 (exenatide; $500 \mathrm{nM}$ ) co-incubated with various concentrations of $\mathrm{E} 4 \times 12-\mathrm{Cy} 7\left(25\right.$ to $100 \mathrm{nM}$ ) for $90 \mathrm{~min}$ at $37^{\circ} \mathrm{C}$. Following incubation, the cells were washed once with $200 \mu \mathrm{L}$ PBS and fixed by adding $100 \mu \mathrm{L}$ of $4 \%$ PFA and allowed to sit at room temperature for 15 min. PFA was removed and washed with PBS $(200 \mu \mathrm{L}, 3 \times)$. The cells were mounted on PBS with NucSpot 488 and the cells were imaged with confocal fluorescence microscope (Leica TCS SP8). 
Tissue mimicking phantom preparation and experimental setup. A phantom mold was prepared to fit the MSOT holder. The phantom allows for a flow-mediated setup as previously shown(35). Briefly, a clear, cylindrical hollow membrane tubing was placed at the center of a cylindrical mold ( $20 \mathrm{~mL}$ syringe, diameter: $2 \mathrm{~cm}$, length: $14 \mathrm{~cm})$. The softtissue mimicking phantom was freshly prepared by adding $15 \% \mathrm{v} / \mathrm{v}$ intralipid $\circledast 20 \%$, I.V. fat emulsion (to provide the scattering), and $0.01 \mathrm{mM}$ Direct Red 81 (for absorption) to a pre-warmed solution of $5 \% \mathrm{v} / \mathrm{v}$ agarose Type 1 (solid in $<37^{\circ} \mathrm{C}$ ) in Milli $\mathrm{Q}$ water (18.2 $\mathrm{M} \Omega$ $\mathrm{cm}^{-1}$ at $25^{\circ} \mathrm{C}$ ). At $15 \%$ agarose, the phantom was suspended in water with the weight of the hollow tubing being supported. The mixture was poured into the mold and allowed to cool and solidify for at least 2 hours and up to overnight at $4{ }^{\circ} \mathrm{C}$. At each end of the tubing, a luer-lock was fitted to connect the syringe pump, equipped with a $20 \mathrm{~mL}$ syringe filled with PBS. Air gaps were used to separate the sample and PBS. The flow rate was kept at $1 \mathrm{~mL} \mathrm{~min}{ }^{-1}$ until the sample of interest reached the region of interest (ROI) for imaging. After imaging, the flow rate was increased $2 \times$ and the phantom was washed with at least $10 \mathrm{~mL}$ PBS. The wash, load sample and wash cycle were repeated for all samples. The flow-mediated phantom setup sustained several acquisitions throughout the day and the same phantom setup was used for all samples that were compared to each other.

Multi-spectral optoacoustic tomography (MSOT). A multi-spectral optoacoustic tomography (MSOT) inVision device (MSOT inVision 256, iThera Medical, Munich, Germany) for small animal imaging was used. MSOT inVision has an array of 256 detector elements that are cylindrically focused, with a central ultrasound frequency of $5 \mathrm{MHz}$ and up to $270^{\circ}$ coverage. The soft-tissue mimicking phantoms were aligned so 
that the illumination ring coincided with the detection plane, i.e. the curved transducer array was centered around the phantom. Data were obtained in the wavelength range of $680-900 \mathrm{~nm}(10 \mathrm{~nm}$ increments) and an average of 10 frames per wavelength were acquired, which equates to $1 \mathrm{~s}$ acquisition time per wavelength per section. The optical excitation originates from a Q-switched Nd:YAG laser with a pulse duration of $10 \mathrm{~ns}$ and a frequency of $10 \mathrm{~Hz}$. Light is homogenously delivered to the phantom using a fiber split into 10 output arms. The fiber bundle and the transducer array are stationary, and the sample holder moves along the z-direction allowing longitudinal acquisition of different imaging planes using a moving stage. MSOT measurements were performed in a temperature-controlled water bath at $34^{\circ} \mathrm{C}$. All variable parameters such as optoacoustic gain, laser power, focus depth, frame averaging, frame rate and high/low pass filters were kept constant during the measurement. Samples were allowed to equilibrate for a minimum of $5 \mathrm{~min}$ before initiating the optoacoustic scan, and concentrations of E4x12Cy7 were from $2 \mu \mathrm{M}, 5 \mu \mathrm{M}, 10 \mu \mathrm{M}, 15 \mu \mathrm{M}$ and $25 \mu \mathrm{M}$ in $0.9 \%$ sodium chloride. Sample solutions were delivered to the center of the phantom using a syringe pump. Between the measurements, the flow device was washed with deionized water $(>10 \mathrm{~mL})$.

Optoacoustic image data processing. Spatial reconstruction and multi-spectral processing (MSP) of the data was performed using the ViewMSOT software suite (V3.6; iThera Medical) and a backprojection algorithm. The normalized optoacoustic spectra were obtained from optoacoustic phantom scans taken from a $25 \mu \mathrm{M}$ sample and used as a contrast agent reference. For multi-spectral unmixing, a linear regression method was used and excluded pixels where the number of wavelengths ( $>25 \%)$ fell within the 
negative signal. Hemoglobin $\left(\mathrm{HbO}_{2}\right)$ and deoxyhemoglobin $(\mathrm{Hb})$ reference spectra included in the software package were used for multi-spectral unmixing. MIN6 cells were implanted in the same position and distance from the surface of the skin across animals. Hence, all animals are affected to the same degree by light scattering. Quantitative information was obtained by defining the ROI manually within a 2D MSOT image. For spectrally unmixed $\mathrm{E} 4 \times 12-\mathrm{Cy} 7$, the mean pixel intensities per cross-section in the volume of interest (VOI) were first plotted as classical least square (CLS) score vs. position (mm) to assess its signal strength. Signal strength is decreasing with distance from the xenograft and creating a parabolic shape. The maximum "mean signal per cross-section" was used as a quantitative indicator of the probe binding. Overall optoacoustic intensities (a.u.) were reported at all imaging wavelengths $(680-900 \mathrm{~nm}, 10 \mathrm{~nm}$ step resolution). For all cases, unless otherwise stated, the CLS method for spectral deconvolution was used and reported as CLS scores. The following reference spectra were used: hemoglobin $\left(\mathrm{HbO}_{2}\right)$, deoxyhemoglobin $(\mathrm{Hb})$ and $\mathrm{E} 4 \times 12-\mathrm{Cy} 7$. To compare the direct intensities of all images, the optoacoustic spectrum $(25 \mu \mathrm{M})$ were normalized to their maximum optoacoustic $(760 \mathrm{~nm})$ wavelength. The channel for $\mathrm{E} 4 \times 12-\mathrm{Cy} 7$ (green) after multi-spectral unmixing was overlaid with the overall optoacoustic intensities (a.u., bone scale).

Animal studies. All animal experiments were performed in accordance with protocols approved by the Institutional Animal Care and Use Committee (IACUC) of Memorial Sloan Kettering Cancer Center (MSK) and followed the National Institutes of Health guidelines for animal welfare. Healthy Hsd:athymic female mice NudeFoxn $1^{\text {nu }}$ (6-8 weeks old) were obtained from the Jackson Laboratory. The mice were split into two groups of three mice, 
one group injected with the imaging agent and the other used a non-injected control group. All animal procedures, other than tail vein injections, were performed with the animals under general $1.5-2 \%$ isoflurane inhalation anesthesia. In order to test GLP-1R receptor specificity, xenografts were created using the pancreatic mouse insulinoma line MIN6. MIN6 cells were injected into the right flank $\left(1 \times 10^{6}\right.$ cells in $50 \mu \mathrm{L} 1: 1 \mathrm{HG}$ DEM medium and Matrigel). The xenografts were left for 12-15 days to allow for proper vasculature formation around the $ß$-cell clusters prior to imaging. From MSOT imaging, the mice were placed into the animal holder in supine position, gently fixed into position using clear straps and fitted with a breathing mask for anesthesia. Ultrasound colorless gel (approximately 1-2 $\mathrm{mm}$ thick layer) was applied onto the mouse around the region of interest in order to improve acoustic coupling. The animal holder was closed, wrapping the clear plastic membrane around the mouse and air gaps and bubbles in between the membrane and mouse's skin were removed. The animal holder with the mouse positioned inside it was then placed into the imaging chamber, with the animal being aligned with regards to the detection plane (centered within the curved transducer array). MIN6-bearing mice $(n=3)$ were imaged with the MSOT prior to injection. Group 1 mice was injected via tail vein with $100 \mu \mathrm{L}$ of $2.9 \mathrm{mM} \mathrm{kg}^{-1}$ of E4x12-Cy7. The mice were re-imaged at 30 min post injection. E4x12-Cy7 was dissolved in $0.9 \%$ sodium chloride solution. Animals were also imaged in tandem using an IVIS Spectrum (PerkinEImer, Waltham, MA, USA) system for planar fluorescence imaging.

Blocking in vivo experiments. MIN6-bearing mice were split into three groups namely, saline, blocking and E4x12-Cy7 injected groups $(n=3)$. Saline group were injected with 
$100 \mu \mathrm{L}$ of $0.9 \%$ sodium chloride, blocking group was injected with $151 \mu \mathrm{g}$ of exendin- 4 $(100 \mu \mathrm{L}$ of $360 \mu \mathrm{M})$ and allowed to circulate for 30 mins, followed by the injection of 57 $\mu \mathrm{E} \mathrm{E} 4 \times 12-\mathrm{Cy} 7(100 \mu \mathrm{L}$ of $120 \mu \mathrm{M})$ and $\mathrm{E} 4 \times 12-\mathrm{C} y 7$ group was injected with $100 \mu \mathrm{g}$ of $\mathrm{E} 4 \times 12-$ Cy7 $(100 \mu \mathrm{L}$ of $120 \mu \mathrm{M})$. Mice were imaged before and after injections using epifluorescence IVIS Spectrum system and the MSOT.

Ex vivo imaging of tissues. After 30 min post-intravenous injection and optoacoustic imaging, kidney, liver, pancreas, spleen, muscle and MIN6 cluster were harvested and planar fluorescence imaging was carried out.

Statistical analysis. Paired t-tests were performed to determine statistical difference in vivo before and after injection. Levels of significance are as follows: ns = not significant, ${ }^{*} p<0.05,{ }^{* *} p<0.01,{ }^{* * *} p<0.001$ and ${ }^{* * * *} p<0.0001$. Data is presented as mean $\pm S D$.

\section{RESULTS}

Synthesis and analysis of E4x12-Cy7. A GLP-1R targeted peptide, exendin-4, was modified at the $\mathrm{K}_{12}$ position of the amino acid sequence with the unnatural alkyne-amino acid (S)-2-amino-4-pentynoic acid (Figure 1A). As reported previously $(4,24,26)$, it can be used without significant reduction of peptide/GLP-1R target binding $(\sim 3 \mathrm{nM})(4)$. Likewise, we have previously shown that cyanine dyes such as Cy7 exhibit the desired optical characteristics suitable for both optoacoustic and fluorescent imaging(36). The synthesis of E4x12-Cy7 was achieved in a one-step reaction (Figure 1B). Cy7-azide (Lumiprobe) was used for labeling of the neopeptide E4×12 (grey) via a bioorthogonal copper(I)- 
catalyzed azide-alkyne cycloaddition reaction (CuAAC) to obtain E4x12-Cy7 (Figure 1B, bottom) in good yield $(>90 \%)$ and purity $\left(78 \%, t_{R}=12.15 \mathrm{~min}\right.$, Figure $\left.1 \mathrm{C}\right)$. The targeting peptide exendin-4, neopeptide $\mathrm{E} 4 \times 12$, and optoacoustic agent $\mathrm{E} 4 \times 12-\mathrm{Cy} 7$ are amino acid sequences consisting of 39 amino acids (Figure 1A). E4×12-Cy7 has a broad absorption, ranging from 550 to $850 \mathrm{~nm}$, with a maximum absorption at $760 \mathrm{~nm}$, indicating an appropriate wavelength range for multi-spectral optoacoustic unmixing(37). Mass spectrometry confirmed the identity of E4×12-Cy7 (Figure 1D).

Validation of E4x12-Cy7 as an optoacoustic agent in tissue-mimicking phantom. The multi-spectral optoacoustic tomography (MSOT) device (MSOT inVision 256, iThera Medical) was used to measure optoacoustic signals. A flow device, fitted into the commercially available MSOT imaging system and containing a soft-tissue mimicking phantom, was prepared as previously described in detail $(35,36)$. In order to evaluate the optoacoustic performance of $\mathrm{E} 4 \times 12-\mathrm{Cy} 7$, solutions of $\mathrm{E} 4 \times 12-\mathrm{Cy} 7$ in $\mathrm{DMSO}$ at concentrations between $2 \mu \mathrm{M}$ and $25 \mu \mathrm{M}$ were prepared. Using a syringe pump, the samples were allowed to flow at roughly $1 \mathrm{~mL} / \mathrm{min}$ until they reached the middle of the phantom. The observed optoacoustic spectrum of E4x12-Cy7 is broad at all of the measured concentrations (Figure 2A, left), with optoacoustic maxima at $760 \mathrm{~nm}$ (Figure 2A, middle), in agreement with the absorbance maxima at $760 \mathrm{~nm}$ (Figure 1C). The optoacoustic spectrum at $25 \mu \mathrm{M}$ was used as the reference spectrum for MSOT spectral unmixing. After spectral unmixing, there was a linear correlation between the optoacoustic signal and concentration of E4x12-Cy7 $\left(R^{2}=0.99\right.$, Figure $2 A$, right). Representative $2 \mathrm{D}$ optoacoustic images of the phantom with the $\mathrm{E} 4 \times 12-\mathrm{Cy} 7$ at varying concentrations were 
reconstructed, and $900 \mathrm{~nm}$ was chosen as the representative optoacoustic background (Figure 2B). A linear regression fitting algorithm using classical least square of known spectra was used to unmix the raw optoacoustic data (a.u.), and the algorithm produces a CLS score(38-40). In our case, three references were included and used for spectral unmixing of hemoglobin, deoxyhemoglobin and $\mathrm{E} 4 \times 12-\mathrm{Cy} 7$.

Binding, affinity and inhibition. Cell assays were performed using MIN6 cells and NIT1 cells, both were derived from mouse pancreatic $ß$-cell insulinomas. E4×12-Cy7 is both an optoacoustic agent and a fluorescent agent, with an $\mathrm{IC}_{50}$ of $4.6 \pm 0.8 \mathrm{nM}$ (Figure $3 \mathrm{~A}$ ). In general, concentrations lower than $50 \mathrm{nM}$ produced the best results, likely due to selfquenching of the tracer at higher concentrations. Incubation of E4×12-Cy7 below $50 \mathrm{nM}$ concentration regimes using MIN6 cells showed a relative increase in fluorescence with increasing concentrations (Figure S1A). Incubation of E4×12-Cy7 at $50 \mathrm{nM}$ in the MIN6 cell line proved to be the optimal concentration for fluorescence imaging (Figure 3B). To test the specificity of E4x12-Cy7 for GLP-1R, we co-incubated MIN6 cells with non-fluorescent exendin-4 (500 nM) and varying concentrations of E4x12-Cy7 (Figure S1B). Co-incubation of exendin-4 (500 nM, 5-20-fold excess) relative to E4x12-Cy7 produced no fluorescence uptake within the cells (Figure S1B). These results showed that E4×12-Cy7 was taken up specifically via by GLP-1R and that its uptake could be inhibited completely by the presence of excess unlabeled peptide. Binding of E4x12-Cy7 to the GLP-1R induced internalization resulting in cytosolic as well as membrane localization at 90 min incubation. 
GLP-1R mediated accumulation of E4x12-Cy 7 . After MSOT pre-imaging sessions, mice used for blocking experiments were injected with $151 \mu \mathrm{g}$ of exendin-4 $(100 \mu \mathrm{L}, 360 \mu \mathrm{M}$, 30 min time interval) before i.v. injection with $57 \mu \mathrm{g} \mathrm{E} 4 \times 12-\mathrm{Cy} 7(100 \mu \mathrm{L}, 120 \mu \mathrm{M}, 30 \mathrm{~min}$ time interval) and MSOT imaging was performed. Likewise, mice that were injected with saline or $57 \mu \mathrm{g}$ of $\mathrm{E} 4 \times 12-\mathrm{Cy} 7$ were imaged before and 20 min after injections. Ex vivo biodistribution via fluorescence imaging showed that the kidney has the highest accumulation of $\mathrm{E} 4 \times 12-\mathrm{Cy} 7$ (Figure S2). A region of interest (ROI) drawn around the kidney before and after MSOT in vivo imaging (Figure 4A) showed that the optoacoustic spectral profile matched that of $\mathrm{E} 4 \times 12-\mathrm{Cy} 7$ (Figure $2 \mathrm{~A}$ left/right). As a reference point, a $\mathrm{ROI}$ around an area that is not kidney was also plotted as background. Therefore, there can be no doubt that the signal after spectral unmixing was from the Cy7 dye. When imaging the kidneys ex vivo with MSOT, a statistically significant difference between saline injected mice and E4x12-Cy7 was observed $\left(p^{*}=0.0178\right.$, Figure S3). To confirm further, the organs from the pancreas, muscle and insulinoma MIN6 were immediately harvested and imaged with the MSOT (Figure 4B). A statistically significant difference between muscle and the pancreas $\left(\mathrm{p}^{* * * *}<0.0001\right)$ and between the muscle and MIN6 $\left(\mathrm{p}^{* * * *}<0.0001\right)$ were observed (unpaired t-test). In addition, in vivo epifluorescence imaging showed statistically significant difference between the blocking and E4x12-Cy7 groups $\left(\mathrm{p}^{* *}=\right.$ 0.0042), showing that the signal was blockable (Figure 4C). Moreover, organs (insulinoma MIN6, pancreas, muscle, kidney, spleen and liver) were excised and placed side-by-side on a black non-fluorescent paper. Epifluorescence imaging was carried out, ROls were drawn around the edges of the organs, and the average fluorescence signal 
was calculated. The signal originating from the mouse insulinoma MIN6 was statistically significant $\left(p^{*}=0.0199\right)$ between the blocking and E4x12-Cy7 groups (Figure 4D).

Optoacoustic imaging of insulinoma. MIN6 cells were used in a mouse model of pancreatic insulinoma because they closely resemble pancreatic ß-cells, including their GLP-1R expression, and insulin secretion $(37,41)$. To determine specific optoacoustic detection and optoacoustic imaging of insulinoma xenografts in vivo, mice were intravenously injected with $57 \mu \mathrm{g} 4 \times 12-\mathrm{Cy} 7 . \mathrm{MSOT}$ imaging of the same mice before injection served as the negative control. Optoacoustic imaging using the MSOT before and 30 min after administration showed a statistically significant increase in signal within the tumors (Figure $5 \mathrm{~A})$. Mice that were imaged 30 min after intravenous injection of $E 4 \times 12^{-}$ Cy7 had an optoacoustic signal score of $72.69 \pm 2.72(n=3$ mice). Pre-injected mice had an optoacoustic background signal score of $30.54 \pm 1.80$ ( $n=3$ mice; Figure 5B). Optoacoustic signal showed a statistically significant difference in mouse insulinoma signal before and after intravenous injection of $E 4 \times 12-C y 7\left({ }^{*} p=0.0236\right)$. The signal strength ratio between the injected and non-injected mice was found to be 2.38 (Figure 5B). In addition, fluorescence imaging in vivo and ex vivo was carried out (Figure S2). Fluorescence imaging corroborated our optoacoustic imaging observations. Ex vivo analysis of E4x12-Cy7 showed uptake in the kidney and liver as well (Figure S2).

\section{DISCUSSION}

Optoacoustic technologies are quickly gaining increasing relevance in diagnostics, tracking disease progression, as well as evaluating treatment therapies through imaging 
of disease-relevant biological processes(42). For this application, the most attractive feature of MSOT over other optoacoustic systems is its ability to provide molecular information, including a range of wavelength measurements in the NIR region that enable multi-spectral unmixing between endogenous and exogenous contrast agents. Hence, a highly absorbing NIR agent, such as our E4x12-Cy7, can achieve sufficient contrast for in vivo ß-cell detection. Compared to traditional fluorescence imaging systems, the key difference of MSOT is that its ability to use light for excitation and sound for detection enables a greater penetration depth. The development of optoacoustic imaging tools for diabetes is a valuable but unmet clinical need $(7,8,25,31,43)$. A handful of proof-ofconcept studies in the literature have emerged to show the potential of diabetes monitoring via optoacoustic imaging, but none feature direct visualizations of ß-cells(6$9,19,44-46)$.

Recent studies typically demonstrated an indirect biological response to the symptoms of diabetes via vasculature tracking $(44,47)$, diabetic neuropathy $(48)$ or glucose sensing $(8,45,46)$. And while CT, MRI, ultrasonography and intravital microscopy(4) are well established in the field of preclinical diabetes imaging, the techniques are hampered by photon absorption and scattering in tissues. Here, an optoacoustic agent E4×12-Cy7 was successfully synthesized, purified, chemically and optoacoustically characterized and validated in vitro and in vivo. A promising sonophore has a high extinction coefficient and broad absorption profile(36), which initially led to our rationale behind Cy7 as the absorber of choice for conjugating to neopeptide $E 4 \times 12$. The introduction of an optoacoustic tracer, Cy7, to the $12^{\text {th }}$ position of the amino acid sequence did not perturb 
its binding affinity to GLP-1R, similar to what we found earlier when substituting at the $14^{\text {th }}$ position $(4,49)$. E4x12-Cy7 maintained its high receptor binding and cellular internalization at 90 min post-incubation and proved to be receptor specific, since it was blocked by $5-20$ fold excess exendin- 4 .

For in vivo imaging, we chose GLP-1R-expressing mouse insulinoma MIN6, derived from transgenic mice and preserving normal characteristics and function of the endocrine $ß-$ cells(37). In vivo, healthy 6-8-week-old Fox $1^{\mathrm{n}}$ nude MIN6-bearing mice were injected with E4x12-Cy7. Intravenous injection of E4x12-Cy7 showed specific uptake after 30 mins, which we were able to detect with both MSOT and fluorescence imaging in the same animal. Cy7 has a broad optoacoustic spectra with a maximum at $760 \mathrm{~nm}$. A similar optoacoustic spectral profile was obtained in the kidney regions after i.v. injection, confirming the presence of E4x12-Cy7 in vivo. It was further corroborated with ex vivo MSOT imaging of the corresponding organs of interest. This signal was blockable with exendin- 4 . To test $\mathrm{E} 4 \times 12-\mathrm{C} y 7$, in vivo imaging was performed in mice with subcutaneous MIN6 insulinomas. This model was chosen to focus to limit biological variability, reducing the complexity that comes with imaging the pancreas. Initially, the visualization of a solid subcutaneous mass of $ß$-cells is much easier than the visualizations of the $1-2 \% ß$-cells that are spread throughout the pancreas. Moreover, we are minimizing the physiologic challenges that exists when imaging mouse pancreata. A mouse pancreas is rather flat and dendritic-like while in humans, it is a well-defined solid mass. Nevertheless, we have also demonstrated uptake in the pancreas. This is expected since approximately $2.4 \times 10^{5}$ B-cells are found in the pancreas of a 10-week old mouse(50) and we injected $1 \times 10^{6} \mathrm{MIN6}$ cells. This may 
be a reason for the signal uptake in the pancreas to appear as high as subcutaneous MIN6 model. From our model, we have observed rapid clearance after 30 min post i.v. injection of E4x12-Cy7 (Figure S4), possibly due to the short half-life (1-2 minutes) of GLP1 caused by N-terminal degradation by the enzyme dipeptidyl peptidase-4 (DPP-4)(51). Although the high extinction coefficient of Cy7 dye $\left(206,000 \mathrm{M}^{-1} \mathrm{~cm}^{-1}\right)$ is adequate for optoacoustic imaging, we suspect its fluorescent nature may not be optimal as an imaging agent for MSOT. Taken together, this could potentially be a limitation and leaves room for further development of other optoacoustic ß-bell targeting agents.

Exendin-4 allows for modular design without perturbing its affinity to GLP-1R. Here, we have shown the first development and in vivo validation of a NIR MSOT agent, E4×12-Cy7, an exedin-4 analog that binds to GLP-1R. Shown in our in vitro and in vivo blocking studies using native peptide exendin-4, we show that the modified sonophore had retained its targeting profile of exendin-4. The MSOT can achieve multi-spectral unmixing methods which delivers information specific from E4x12-Cy7. Consequently, E4x12-Cy7 could work well with the hand-held optoacoustic imaging system. In addition, the incorporation of 3D optoacoustic tomographic systems could work in favor with near infrared absorbers, known to provide more quantitative assessments(30). Based on the previous success of specific ß-cell imaging agents for PET, fluorescence imaging or in combinations thereof $(4,24,26)$, we envision that a contrast agent such as E4x12-Cy7 could represent a potential platform for further development and optimization of ß-cell optoacoustic imaging. 


\section{CONCLUSION}

In this study, we synthesized and characterized $\mathrm{E} 4 \times 12-\mathrm{Cy} 7$, an optoacoustic imaging agent based on exendin-4, a first generation GLP-1R-targeted optoacoustic ß-cell agent. Using MSOT, E4x12-Cy7 showed specific uptake for MIN6 xenografts. The high spatial resolution and penetration depth achieved with MSOT, in combination with targeted NIR E4x12-Cy7, could represent a valuable technique for monitoring diabetes and its progression in both preclinical and clinical contexts. This study is the first step towards the development of ßcell mass optoacoustic imaging, a technique that merits further investigation as the already enormous challenge diabetes represents continues to grow.

Acknowledgements. The authors thank the support of Memorial Sloan Kettering Cancer Center's Animal Imaging Core Facility, Radiochemistry \& Molecular Imaging Probes Core Facility, Molecular Cytology Core Facility, Nuclear Magnetic Resonance Analytical Core Facility and the University of Michigan Flow Cytometry Core Facility. The authors thank Christopher Wittman for assistance in cell culture during the revision of the paper and Garon Scott for proof-reading the manuscript. This work was supported by National Institutes of Health grants R01 CA204441 (T. R.), R01 CA183953 (J.G.), R35 GM128819 (G.M.T.) and P30 CA008748.

Author Contributions. S.R. and T.R. designed the study. S.R. and E.K and T. N. performed experiments. C.C. maintained cell culture work. S.R. and T.R. primarily analyzed, interpreted and wrote the manuscript. J.G. and G. M. T. consulted and edited 
the manuscript. All authors have read, provided feedback on, and approved the manuscript for publication.

Conflict of interests. T.R. is a shareholder of Summit Biomedical Imaging, LLC. J.G. and T.R. are co-inventors on filed U.S. patent (US20190275179A1) which covers optoacoustic imaging agents. T.R. is co-inventor on U.S. patent (WO2012121746A2), covering the composition of matter for islet imaging agents. T.R. is a paid consultant for and has received grant support from Theragnostics, Inc. This arrangement has been reviewed and approved by Memorial Sloan Kettering Cancer Center in accordance with its conflict of interest policies. 


\section{KEYPOINTS}

Question: Is optoacoustic imaging of the glucagon-like peptide-1 receptor (GLP-1 Receptor) possible with a first-generation near infrared exendin-4 analog, E4×12-Cy7?

Pertinent Findings: A novel optoacoustic imaging probe, E4x12-Cy7 targets the GLP-1 receptors on the surface of $ß$-cells in a pancreatic mouse model of insulinoma. E4×12-Cy7 was successfully synthesized and showed high binding affinity comparable to exendin- 4 (exenatide). Multi-spectral optoacoustic tomography (MSOT) with $\mathrm{E}_{\times 12-\mathrm{Cy}} \mathrm{7}$ allowed optoacoustic imaging of GLP-1 receptors in vivo which were blockable.

Implications for patient care: A near infrared E4x12-Cy7 and similar analogs can be exploited for MSOT imaging, combining capabilities of both light for excitation and sound for detection that could prove to be important contrast agents for monitoring the pancreas for the progression of diabetes. 


\section{REFERENCES}

1. Diabetes Association A. 2. Classification and Diagnosis of Diabetes: Standards of Medical Care in Diabetes-2018. Diabetes Care. 2018;41:S13- S27.

2. Wild S, Roglic G, Green A, Sicree R, King H. Global Prevalence of Diabetes: estimates for the year 2000 and projections for 2030. Diabetes Care. 2004;27:1047- 1053.

3. Rowley WR, Bezold C, Arikan Y, Byrne E, Krohe S. Diabetes 2030: Insights from Yesterday, Today, and Future Trends. Population health management. 2017;20:6-12.

4. Reiner $\mathrm{T}$, Thurber $\mathrm{G}$, Gaglia J, et al. Accurate measurement of pancreatic islet $\beta$-cell mass using a second-generation fluorescent exendin-4 analog. Proceedings of the National Academy of Sciences. 2011;108:12815-12820.

5. Reiner T, Kohler RH, Liew CW, et al. Near-infrared fluorescent probe for imaging of pancreatic beta cells. Bioconjugate chemistry. 2010;21:1362-1368.

6. MacKenzie HA, Ashton HS, Spiers S, et al. Advances in Photoacoustic Noninvasive Glucose Testing. Clinical Chemistry. 1999;45:1587- 1595.

7. Dasa MK, Markos C, Janting J, Bang O. Multispectral photoacoustic sensing for accurate glucose monitoring using a supercontinuum laser. Journal of the Optical Society of America $B$. 2019;36:A61-A65.

8. Ghazaryan A, Ovsepian SV, Ntziachristos V. Extended Near-Infrared Optoacoustic Spectrometry for Sensing Physiological Concentrations of Glucose. Frontiers in endocrinology. 2018;9:112-112.

9. Zhang R, Gao F, Feng X, et al. "Guide Star" Assisted Noninvasive Photoacoustic Measurement of Glucose. ACS Sensors. 2018;3:2550-2557.

10. Bruen D, Delaney C, Florea L, Diamond D. Glucose Sensing for Diabetes Monitoring: Recent Developments. Sensors (Basel, Switzerland). 2017;17:1866- 1888.

11. Pattou F, Kerr-Conte J, Wild D. GLP-1-Receptor Scanning for Imaging of Human Beta Cells Transplanted in Muscle. New England Journal of Medicine. 2010;363:1289-1290.

12. Jones B, Buenaventura $\mathrm{T}$, Kanda N, et al. Targeting GLP-1 receptor trafficking to improve agonist efficacy. Nature Communications. 2018;9:1602- 1619.

13. Raptis AE, Markakis KP, Mazioti MC, Raptis SA, Dimitriadis GD. What the radiologist needs to know about the diabetic patient. Insights into imaging. 2011;2:193-203. 
14. Vetere A, Choudhary A, Burns SM, Wagner BK. Targeting the pancreatic $\beta$-cell to treat diabetes. Nature Reviews Drug Discovery. 2014;13:278- 289.

15. van Gurp L, Loomans CJM, van Krieken PP, et al. Sequential intravital imaging reveals in vivo dynamics of pancreatic tissue transplanted under the kidney capsule in mice. Diabetologia. 2016;59:2387-2392.

16. Coppieters $\mathrm{K}$, Amirian N, von Herrath M. Intravital imaging of CTLs killing islet cells in diabetic mice. The Journal of clinical investigation. 2012;122:119-131.

17. Brom $M$, Joosten $L$, Frielink $C$, et al. Validation of 111 In-Exendin SPECT for the Determination of the $\beta$-Cell Mass in BioBreeding Diabetes-Prone Rats. Diabetes. 2018;67:20122018.

18. Luo Y, Pan Q, Yao S, et al. Glucagon-Like Peptide-1 Receptor PET/CT with 68Ga-NOTAExendin-4 for Detecting Localized Insulinoma: A Prospective Cohort Study. Journal of nuclear medicine : official publication, Society of Nuclear Medicine. 2016;57:715-720.

19. Antwi K, Fani M, Nicolas G, et al. Localization of Hidden Insulinomas with 68Ga-DOTAExendin-4 PET/CT: A Pilot Study. Journal of Nuclear Medicine. 2015;56:1075-1078.

20. Normandin MD, Petersen KF, Ding Y-S, et al. In Vivo Imaging of Endogenous Pancreatic $\beta$-Cell Mass in Healthy and Type 1 Diabetic Subjects Using 18F-FluoropropylDihydrotetrabenazine and PET. Journal of Nuclear Medicine. 2012;53:908-916.

21. Carlbom L, Espes D, Lubberink M, et al. [11C]5-hydroxy-tryptophan PET for Assessment of Islet Mass During Progression of Type 2 Diabetes. Diabetes. 2017;66:1286-1292.

22. Bini J, Naganawa M, Nabulsi N, et al. Evaluation of PET Brain Radioligands for Imaging Pancreatic $\beta$-Cell Mass: Potential Utility of 11C-(+)-PHNO. Journal of Nuclear Medicine. 2018;59:1249-1254.

23. Diamant M, Van Gaal L, Stranks S, et al. Once weekly exenatide compared with insulin glargine titrated to target in patients with type 2 diabetes (DURATION-3): an open-label randomised trial. The Lancet. 2010;375:2234-2243.

24. Khera E, Zhang L, Roberts S, et al. Blocking Glucagon Like Peptide-1 Receptors in the Exocrine Pancreas Improves Specificity for Beta Cells in a Mouse Model of Type 1 Diabetes. Journal of Nuclear Medicine. 2019;60:1635-1641.

25. Jodal A, Schibli R, Béhé $M$. Targets and probes for non-invasive imaging of $\beta$-cells. European journal of nuclear medicine and molecular imaging. 2017;44:712-727. 
26. Brand $C$, Abdel-Atti D, Zhang $Y$, et al. In Vivo Imaging of GLP-1R with a Targeted Bimodal PET/Fluorescence Imaging Agent. Bioconjugate Chemistry. 2014;25:1323-1330.

27. Clardy SM, Keliher EJ, Mohan JF, et al. Fluorescent exendin-4 derivatives for pancreatic $\beta$-cell analysis. Bioconjugate chemistry. 2014;25:171-177.

28. Saudek F, Brogren $\mathrm{C}-\mathrm{H}$, Manohar S. Imaging the Beta-Cell Mass: Why and How. The Review of Diabetic Studies : RDS. 2008;5:6-12.

29. Chen C, Cohrs CM, Stertmann J, Bozsak R, Speier S. Human beta cell mass and function in diabetes: Recent advances in knowledge and technologies to understand disease pathogenesis. Molecular metabolism. 2017;6:943-957.

30. Deán-Ben XL, Fehm TF, Ford SJ, Gottschalk S, Razansky D. Spiral volumetric optoacoustic tomography visualizes multi-scale dynamics in mice. Light: Science \& Applications. 2017;6:e16247-e16247.

31. Schellenberg MW, Hunt HK. Hand-held optoacoustic imaging: A review. Photoacoustics. 2018;11:14-27.

32. Knieling F, Neufert C, Hartmann A, et al. Multispectral Optoacoustic Tomography for Assessment of Crohn's Disease Activity. New England Journal of Medicine. 2017;376:1292-1294.

33. Regensburger AP, Fonteyne LM, Jüngert J, et al. Detection of collagens by multispectral optoacoustic tomography as an imaging biomarker for Duchenne muscular dystrophy. Nature Medicine. 2019;25:1905-1915.

34. Neuschmelting V, Burton NC, Lockau H, et al. Performance of a Multispectral Optoacoustic Tomography (MSOT) System equipped with 2D vs. 3D Handheld Probes for Potential Clinical Translation. Photoacoustics. 2016;4:1-10.

35. Roberts S, Strome A, Choi C, et al. Acid specific dark quencher QC1 pHLIP for multispectral optoacoustic diagnoses of breast cancer. Scientific Reports. 2019;9:8550-8562.

36. Roberts $S$, Andreou C, Choi C, et al. Sonophore-enhanced nanoemulsions for optoacoustic imaging of cancer. Chemical Science. 2018;9:5646-5657.

37. Ishihara $\mathrm{H}$, Asano $\mathrm{T}$, Tsukuda $\mathrm{K}$, et al. Pancreatic beta cell line MIN6 exhibits characteristics of glucose metabolism and glucose-stimulated insulin secretion similar to those of normal islets. Diabetologia. 1993;36:1139-1145.

38. Glatz J, Deliolanis NC, Buehler A, Razansky D, Ntziachristos V. Blind source unmixing in multi-spectral optoacoustic tomography. Optics Express. 2011;19:3175-3184. 
39. Tzoumas S, Nunes A, Olefir I, et al. Eigenspectra optoacoustic tomography achieves quantitative blood oxygenation imaging deep in tissues. Nature Communications. 2016;7:12121.

40. Ding L, Deán-Ben XL, Burton NC, Sobol RW, Ntziachristos V, Razansky D. Constrained Inversion and Spectral Unmixing in Multispectral Optoacoustic Tomography. IEEE Transactions on Medical Imaging. 2017;36:1676-1685.

41. Cheng K, Delghingaro-Augusto V, Nolan CJ, et al. High Passage MIN6 Cells Have Impaired Insulin Secretion with Impaired Glucose and Lipid Oxidation. PLOS ONE. 2012;7:e40868 p4086140812.

42. Johnson SP, Ogunlade O, Lythgoe MF, Beard P, Pedley RB. Longitudinal photoacoustic imaging of the pharmacodynamic effect of vascular targeted therapy on tumors. Clinical Cancer Research. 2019:clincanres.0360.2019.

43. Chan DXH, Sugii S, Han W. A UCP1 Near-Infrared Reporter System for Monitoring Adipose "Browning" In Vivo Using Photoacoustic Imaging. Diabetes. 2018;67:280-OR.

44. Krumholz A, Wang L, Yao J, Wang LV. Functional photoacoustic microscopy of diabetic vasculature. Journal of biomedical optics. 2012;17:060502-060501-060503.

45. Sim JY, Ahn C-G, Jeong E-J, Kim BK. In vivo Microscopic Photoacoustic Spectroscopy for Non-Invasive Glucose Monitoring Invulnerable to Skin Secretion Products. Scientific Reports. 2018;8:1059-1070.

46. Weiss R, Yegorchikov Y, Shusterman A, Raz I. Noninvasive Continuous Glucose Monitoring Using Photoacoustic Technology-Results from the First 62 Subjects. Diabetes Technology \& Therapeutics. 2007;9:68-74.

47. Feng W, Shi R, Zhang C, Liu S, Yu T, Zhu D. Visualization of skin microvascular dysfunction of type 1 diabetic mice using in vivo skin optical clearing method. J Biomed Opt. 2018;24:1-9.

48. Yagihashi S. Clinical Application of Photoacoustic Imaging to the Evaluation of Diabetic Polyneuropathy. Diabetes. 2018;67:582-582.

49. Zhang L, Thurber GM. Quantitative Impact of Plasma Clearance and Down-regulation on GLP-1 Receptor Molecular Imaging. Molecular Imaging and Biology. 2016;18:79-89.

50. Chintinne M, Stangé G, Denys B, Ling Z, In 't Veld P, Pipeleers D. Beta cell count instead of beta cell mass to assess and localize growth in beta cell population following pancreatic duct ligation in mice. PloS one. 2012;7:e43959-e43959. 
51. Manandhar B, Ahn J-M. Glucagon-like peptide-1 (GLP-1) analogs: recent advances, new possibilities, and therapeutic implications. Journal of medicinal chemistry. 2015;58:1020-1037. 


\section{FIGURES}

A

\begin{tabular}{ccccc} 
abbreviation & amino acid sequence & length (aa) & NIRF mod & molecular mass (g/mol) \\
\hline exendin-4 & HGEGTFTSDLSKQMEEEAVRLFIEWLKNGGPSSGAPPPS & 39 & none & 4188 \\
\hline E4 412 & HGEGTFTSDLSXQMEEEAVRLFIEWLKNGGPSSGAPPPS & 39 & $\mathrm{X} 12$ & 4155 \\
\hline E4×12-Cy7 & HGEGTFTSDLSX(PEG-CY7)QMEEEAVRLFIEWLKNGGPSSGAPPPS & 39 & $\mathrm{X} 12(\mathrm{Cy})$ & 4786 \\
\hline
\end{tabular}

B

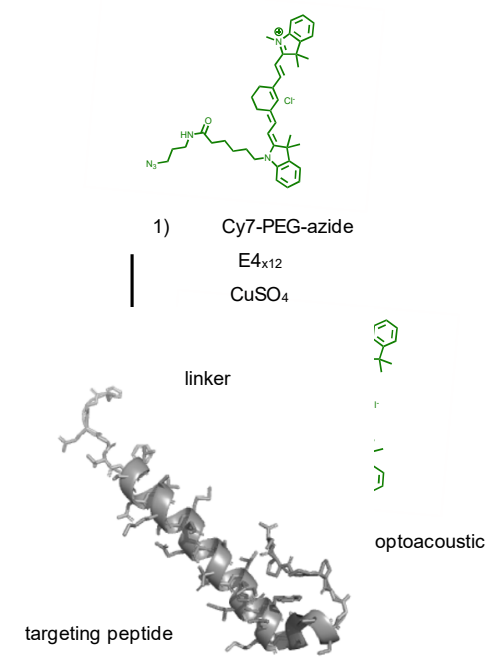

C

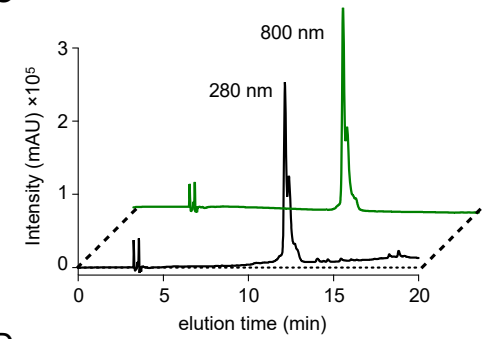

D

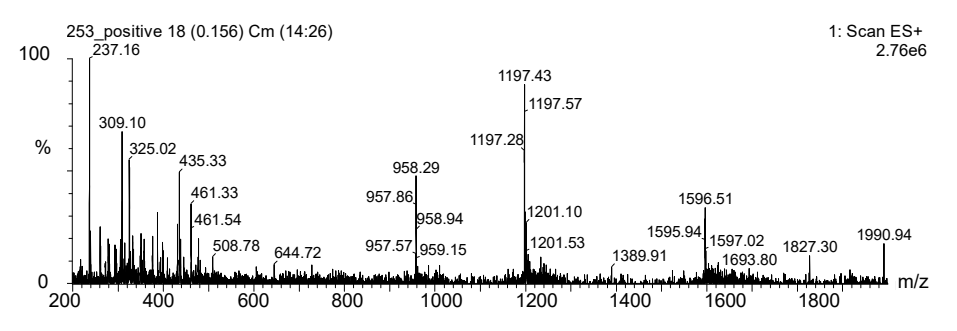

FIGURE 1. Synthesis and analysis of E4x12-Cy7. (A) Abbreviations and amino acid sequences of exendin-4 and its modified derivatives. (B) Copper-catalyzed azide-alkyne cycloaddition reaction scheme yielding optoacoustic probe E4×12-Cy7. (C) HPLC trace, 595\% MeCN, 15 min linear gradient (left) and absorbance/emission spectra (right) of E4×12Cy7. (D) ESI-MS spectrum of E4x12-Cy7. 

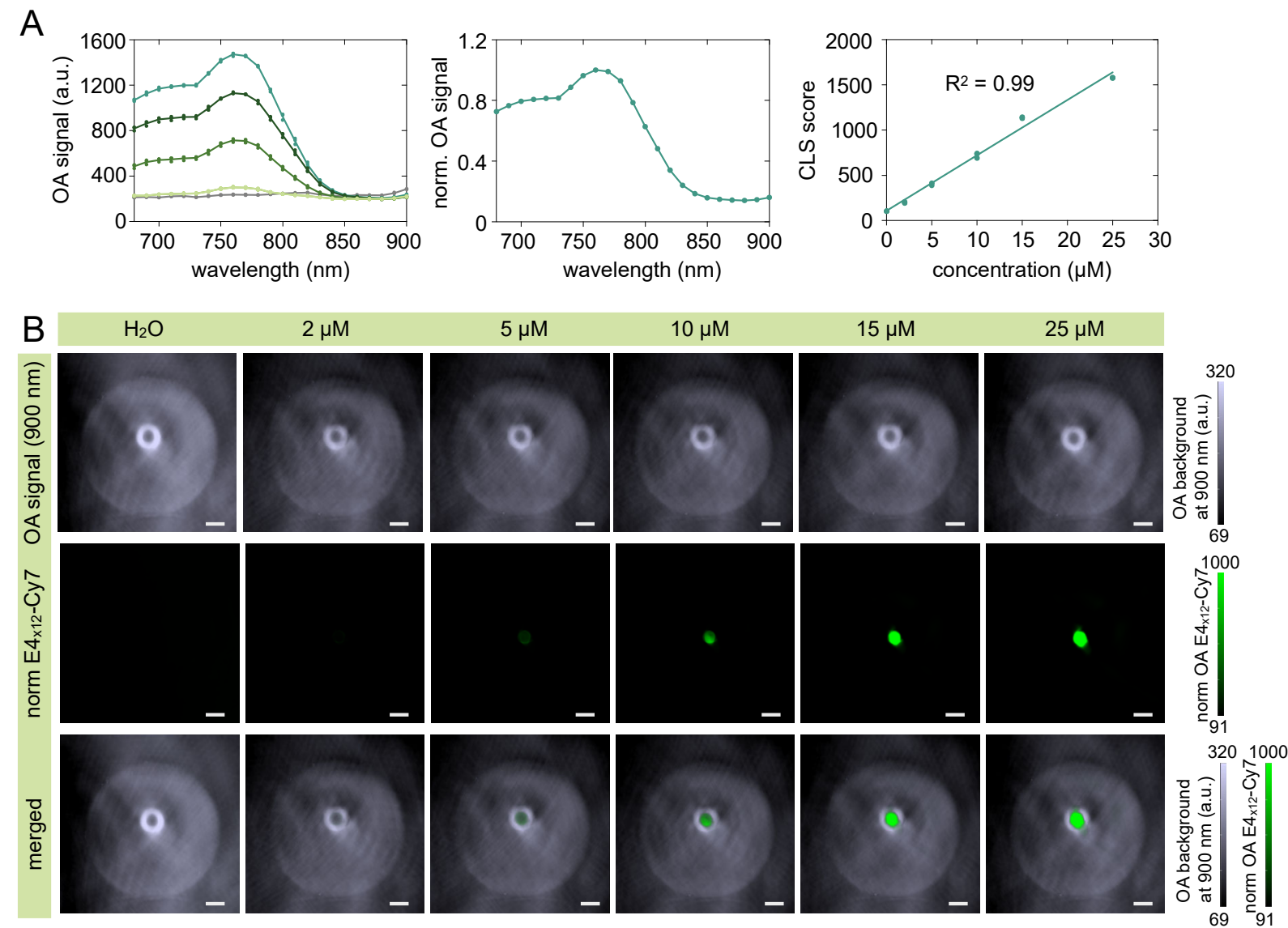

FIGURE 2. Evaluation of pancreatic ß-cell targeting peptide $(E 4 \times 12-C y 7)$ as an optoacoustic probe in soft-tissue mimicking phantoms. (A, left) Optoacoustic spectra from 680-900 nm with $10 \mathrm{~nm}$ wavelength resolution at various concentrations of E4×12-Cy7 (2 $\mu \mathrm{M}, 5 \mu \mathrm{M}, 10 \mu \mathrm{M}, 15 \mu \mathrm{M}$ and $25 \mu \mathrm{M})$. (A, middle) Normalized spectrum $(25 \mu \mathrm{M})$ at the maxima optoacoustic signal. (A, right) Optoacoustic signal at various concentrations obtained after multi-spectral unmixing. (B) Optoacoustic image reconstruction of E4×12Cy7 solutions embedded in a homogenous and absorbing soft-tissue mimicking phantom. White scale bar is $2.5 \mathrm{~mm}$. 
A

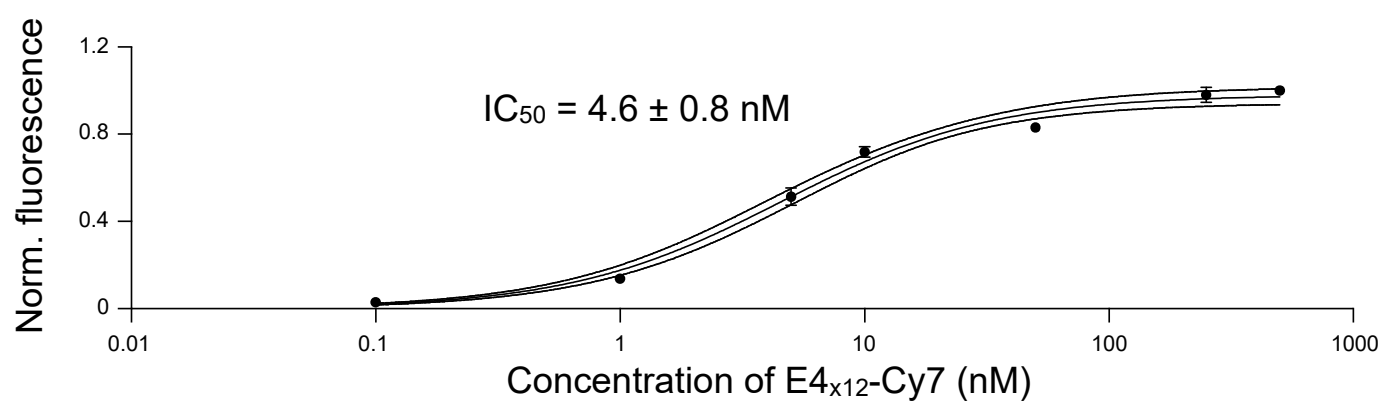

B
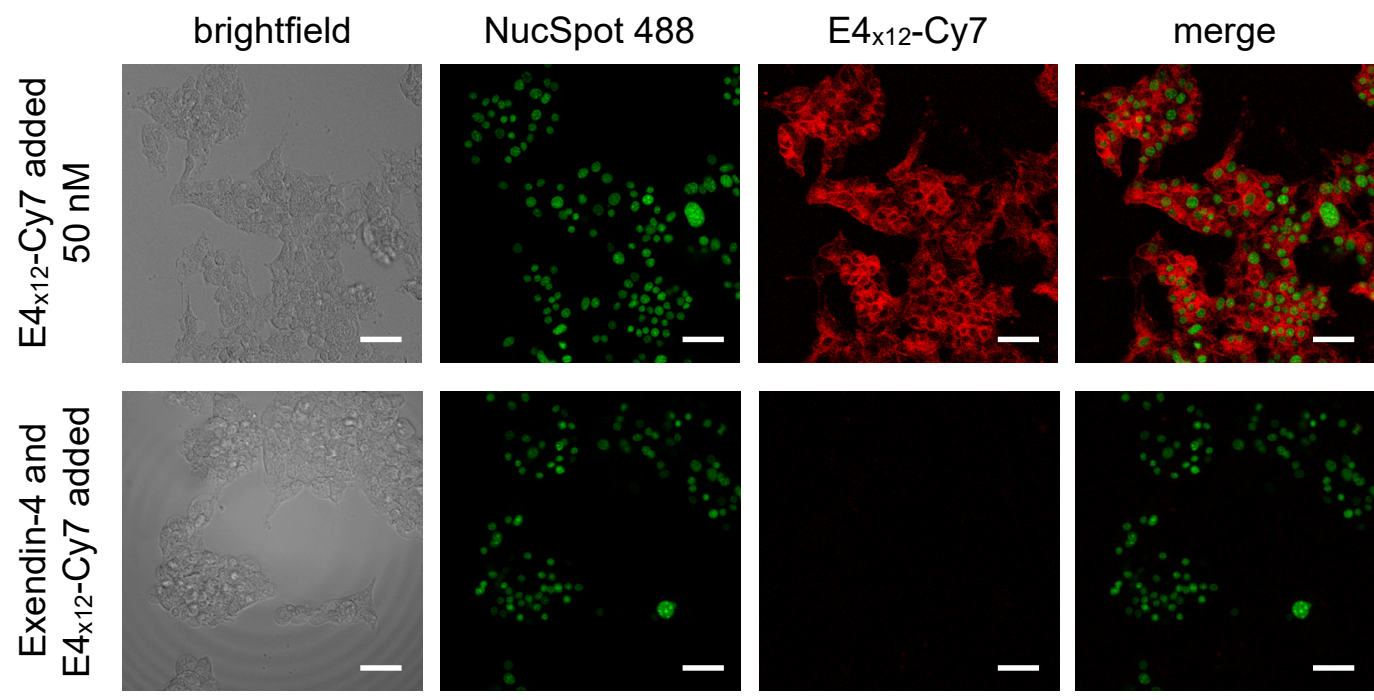

FIGURE 3. In vitro binding and inhibition studies. (top) IC 50 value of $\mathrm{E}_{\mathrm{x} \times 12}-\mathrm{Cy} 7$ measured via a competitive binding assay using NIT-1 cells. (bottom) Confocal microscopy imaging experiments using MIN6 cells following the addition of E4x12-Cy7 (top panel) or after coincubation with excess exendin-4 (lower panel). From left to right is the brightfield image, the image showing nuclear staining with NucSpot 488, and the image showing 50 nM E4x12-Cy7 staining or coincubation with excess exendin-4 and its composite. Scale bars are $40 \mu \mathrm{m}$. 
A
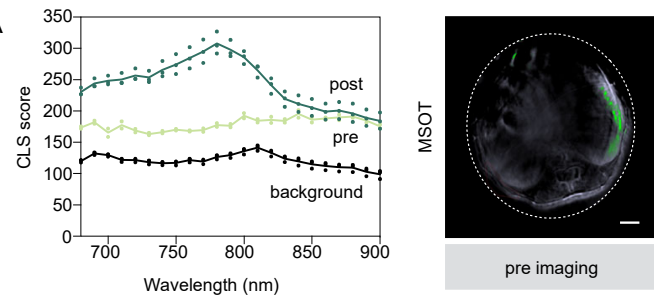

pre imaging
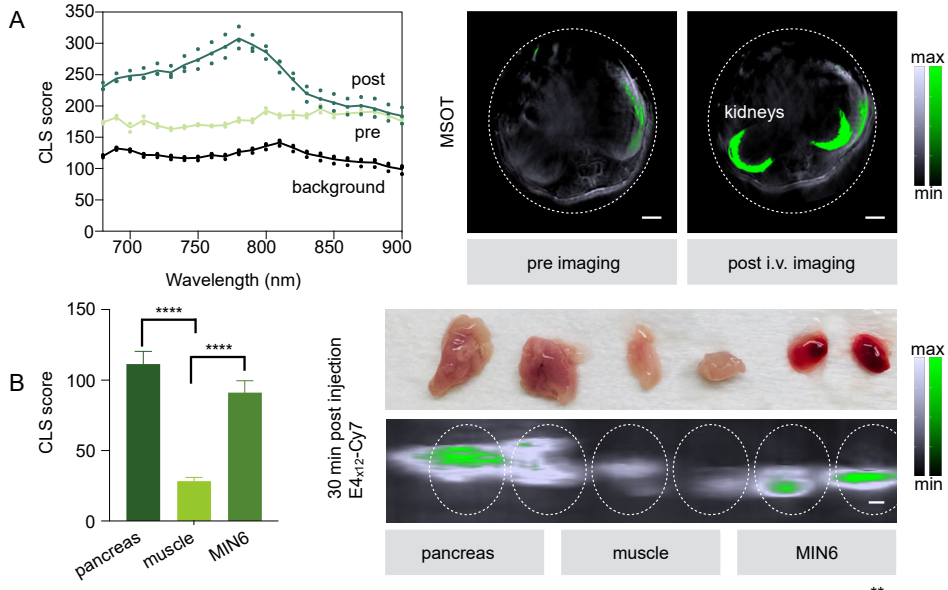

C
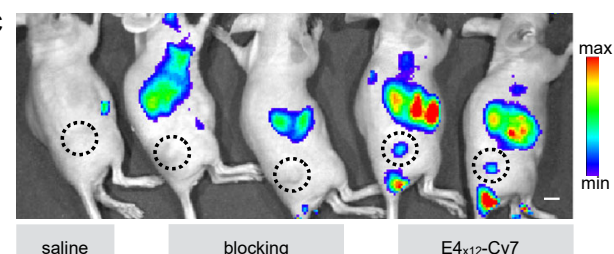

$\mathrm{E} 4_{\times 12}-\mathrm{Cy} 7$
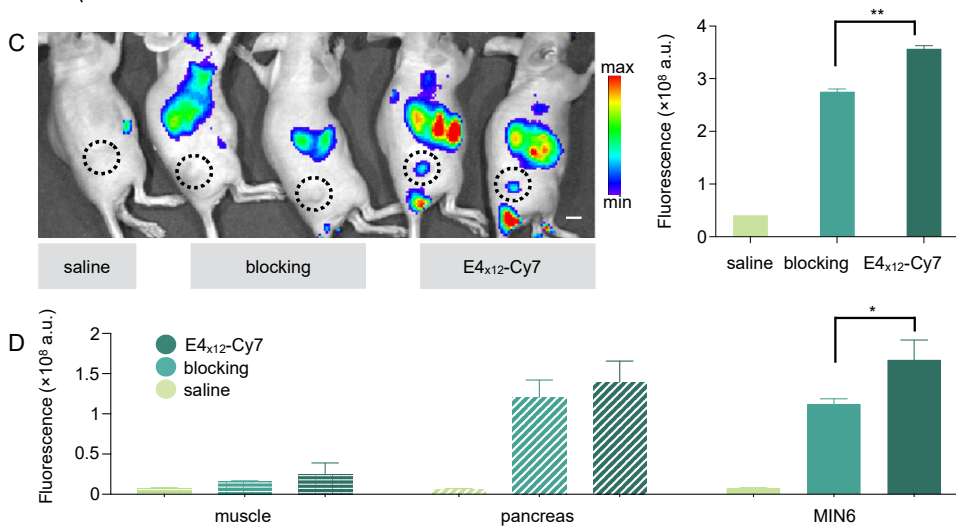

FIGURE 4. The accumulation of E4x12-Cy7 is GLP-1R mediated. (A) Optoacoustic signal at varying wavelengths (680- $900 \mathrm{~nm}, 10 \mathrm{~nm}$ step) taken at the kidney region (left) and the corresponding MSOT images (right). Scale bar is $2.5 \mathrm{~mm}$. (B) Ex vivo MSOT images of organs (pancreas, muscle and MIN6) from mice that were injected with $E 4 \times 12-C y 7$ (right) and the corresponding quantification (left). Scale bar is $2.5 \mathrm{~mm}$. (C) Representative in vivo epifluorescent images of mice that were injected either with saline, a mixture of exendin-4 and $\mathrm{E} 4 \times 12-\mathrm{Cy} 7$ (3:1 ratio) or $\mathrm{E} 4 \times 12-\mathrm{Cy} 7$ (left) and in vivo quantification (right). Scale bar is $0.79 \mathrm{~cm}$. (D) Quantification from epifluorescent images of ex vivo muscle, pancreas and MIN6. The ex vivo organs correspond to mice that were either injected with saline, a mixture of exendin- 4 and $\mathrm{E} 4 \times 12-\mathrm{Cy} 7$ (3:1 ratio) or $\mathrm{E} 4 \times 12-\mathrm{Cy} 7$ (left to right). 

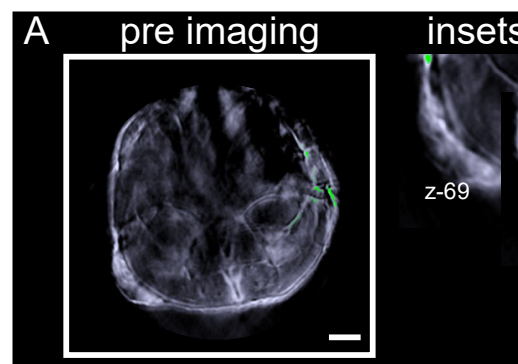

z-69

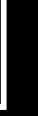

post i.v. imaging

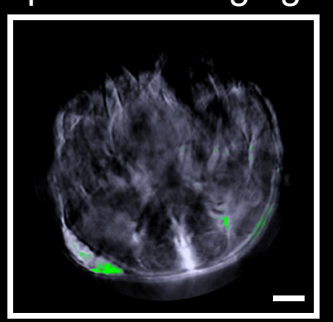

OA background

37

$(680 \mathrm{~nm})$

$5.2 \times 10^{4}$
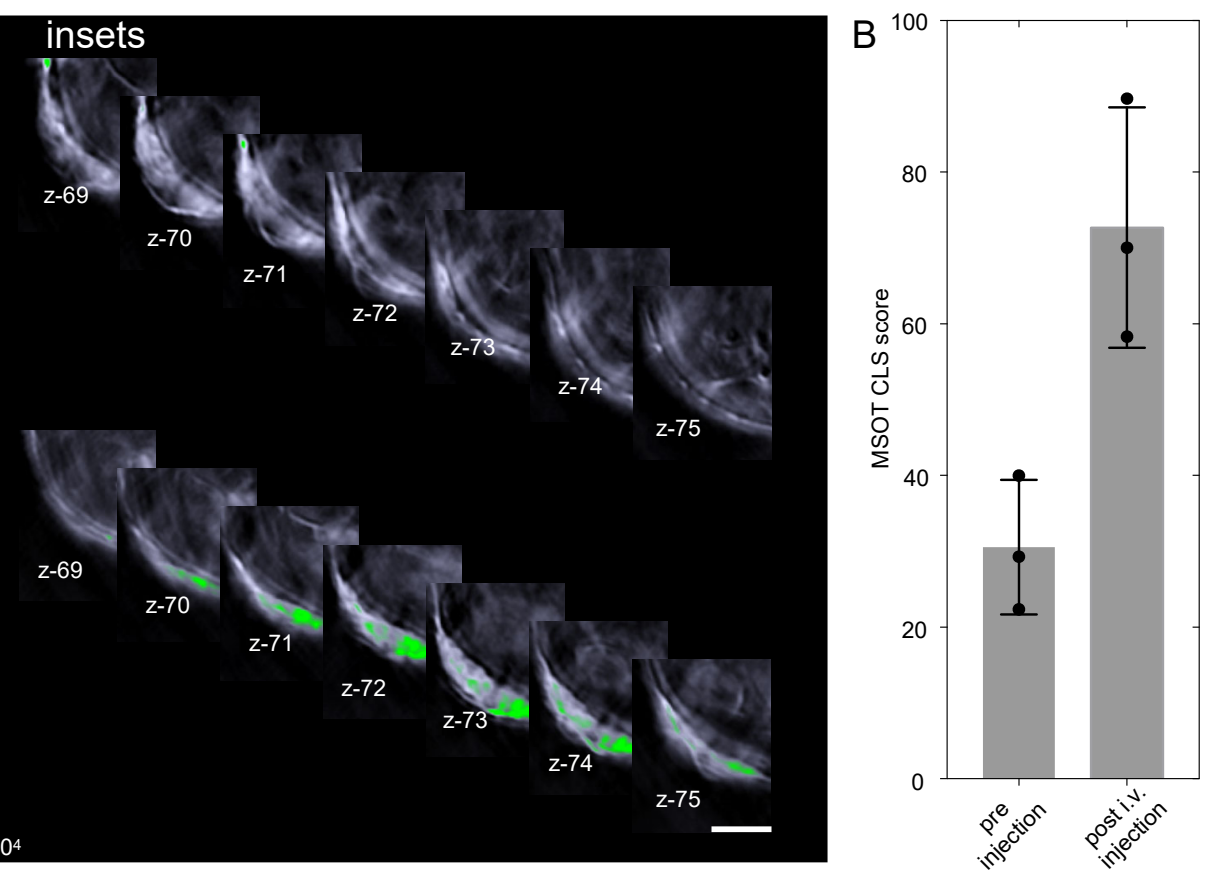

FIGURE 5. In vivo multi-spectral optoacoustic evaluation of E4×12-Cy7. (A) Optoacoustic image reconstruction showing transverse $2 \mathrm{D}$ projection of mice at the region of interest before and $30 \mathrm{~min}$ after intravenous injection of E4x12-Cy7 $(2.9 \mathrm{mg} / \mathrm{kg})$. After multi-spectral unmixing, the E4x12-Cy7 channel (green) was overlaid on top of overall optoacoustic signal at $680 \mathrm{~nm}$ (greyscale). All scale bars are $2.5 \mathrm{~mm}$. (B) Optoacoustic signal quantification after multi-spectral unmixing. 


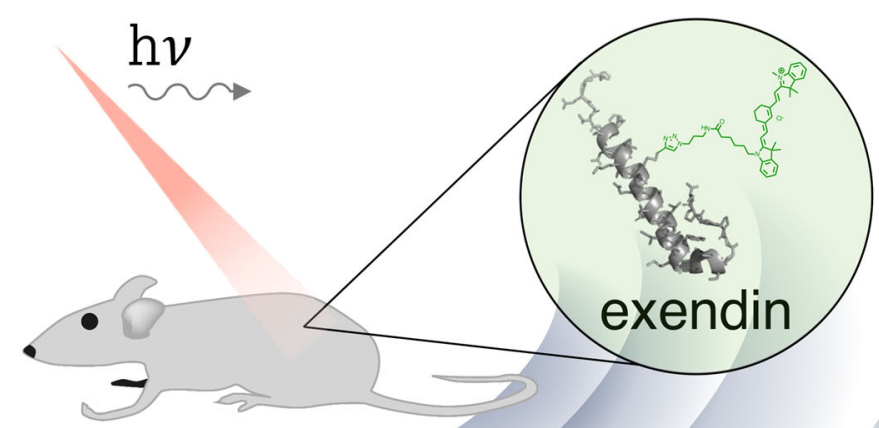

pre post injection

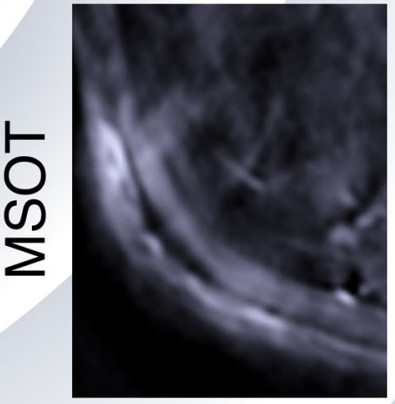

optoacoustic sign

\section{Graphical Abstract}




\section{SUPPLEMENTARY INFORMATION FOR}

\section{Optoacoustic imaging of Glucagon-like Peptide 1 Receptor with a near-infrared exendin-4 analog}

Running title: Optoacoustic imaging of GLP-1R

Sheryl Roberts ${ }^{1}$, Eshita Khera ${ }^{2}$, Crystal Choi ${ }^{1}$, Tejas Navaratna ${ }^{2}$, Jan Grimm ${ }^{1,3,4,5}$, Greg M. Thurber ${ }^{2,6}$, Thomas Reiner $1,4,7 *$

${ }^{1}$ Department of Radiology, Memorial Sloan Kettering Cancer Center, New York, NY, 10065, USA

${ }^{2}$ Department of Chemical Engineering, University of Michigan, Ann Arbor, Michigan, United States

${ }^{3}$ Department of Molecular Pharmacology, Memorial Sloan Kettering Cancer Center, New York, New York, USA

${ }^{4}$ Department of Radiology, Weill Cornell Medical College, 1300 York Avenue, New York, New York.

${ }^{5}$ Pharmacology Program, Weill Cornell Medical College, 1300 York Avenue, New York, New York, 10065, USA

${ }^{6}$ Department of Biomedical Engineering, University of Michigan, Ann Arbor, Michigan, Unites States

${ }^{7}$ Chemical Biology Program, Memorial Sloan Kettering Cancer Center, New York, New York 10065

Correspondence should be addressed to:

*Thomas Reiner

1275 York Avenue

New York, NY, 10065

reinert@mskcc.org; (P) 1-646-888-3461; (F) 6464420408 


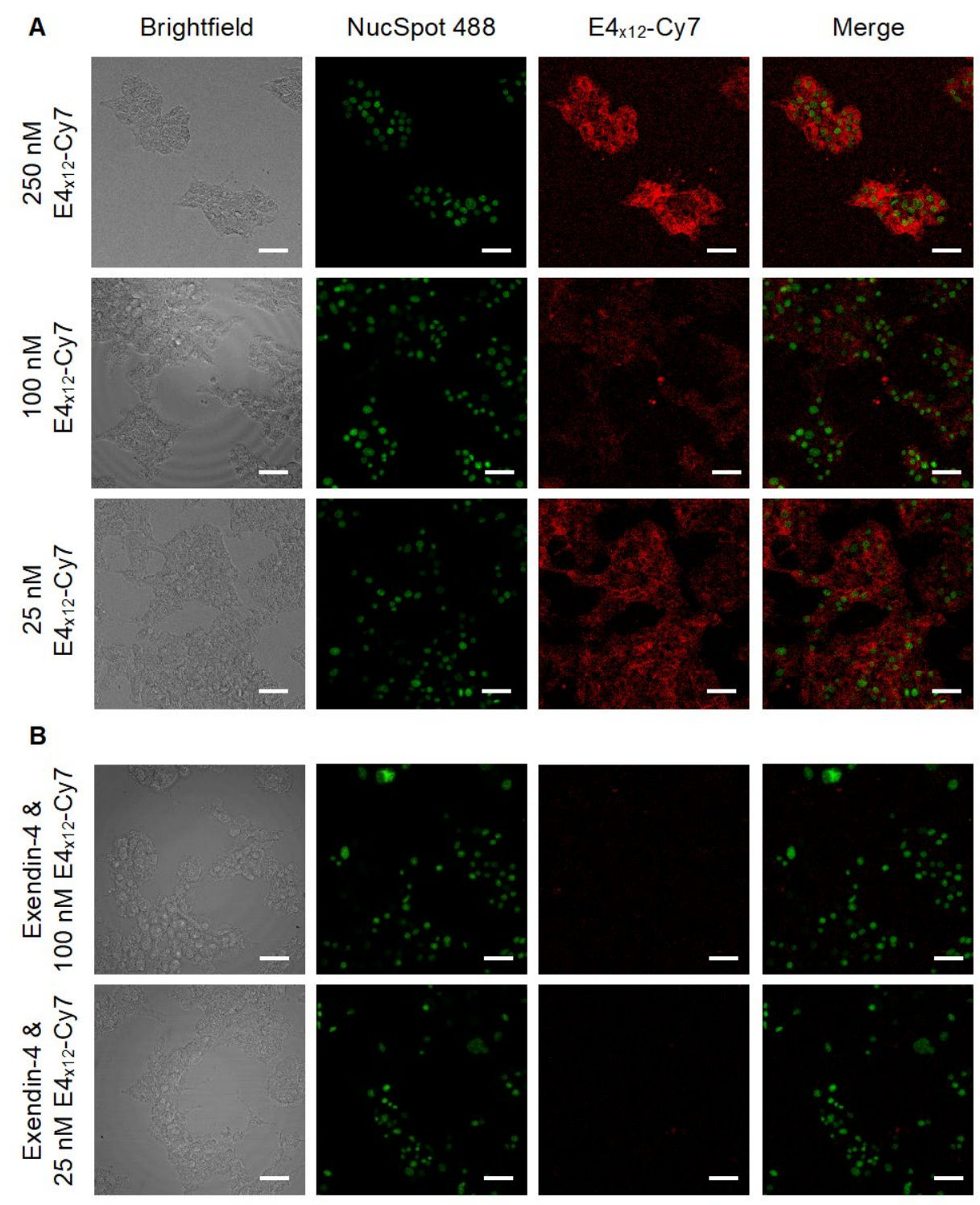

Figure S1. In vitro binding and inhibition studies. (A) Confocal microscopy imaging experiments using MIN6 cells following the addition of E4×12-Cy7 (red) at different concentrations: $250 \mathrm{nM}$ (top row), $100 \mathrm{nM}$ (middle row) and $25 \mathrm{nM}$ (bottom row). Columns from left to right: brightfield ( $1^{\text {st }}$ column), nuclear staining with NucSpot 488 (green, $2^{\text {nd }}$ column), E4x12-Cy7 staining (red, $3^{\text {rd }}$ column) and the corresponding composite image (4 $4^{\text {th }}$ column). (B) Coincubation of E4×12-Cy7 at $100 \mathrm{nM}$ (top) and $25 \mathrm{nM}$ (bottom) with excess exendin-4. Scale bars are $40 \mu \mathrm{m}$. 


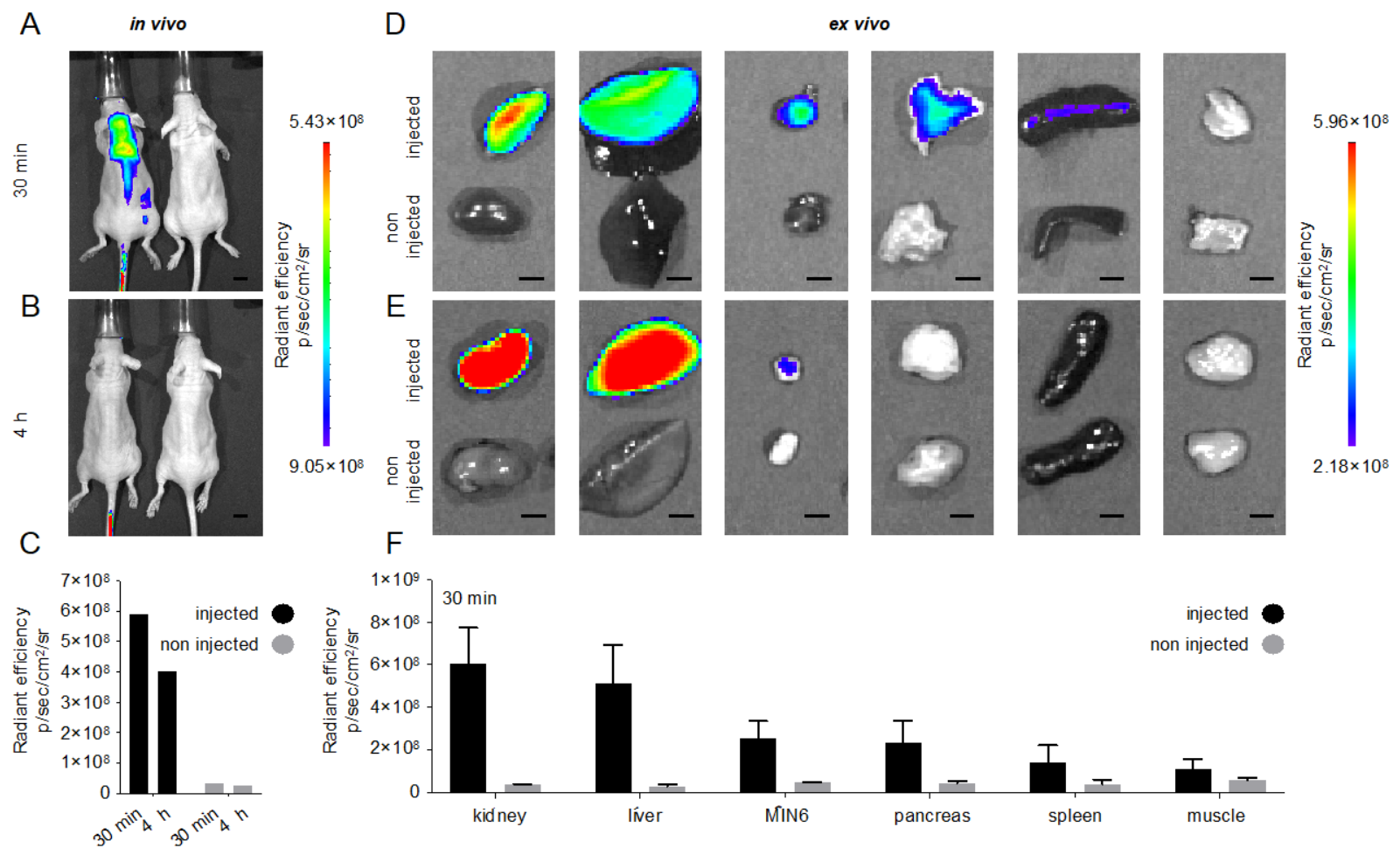

Figure S2. In vivo and ex vivo kinetics and biodistribution validation of E4×12-Cy7 using fluorescence IVIS imaging. (A) Representative in vivo fluorescent images of E4×12-Cy7 30 min post-i.v. injection (left) and vehicle (right), (B) 4 h post-i.v. injection (left) and vehicle (right) and $(C)$ quantifications $(n=3)$. Scale bars in $A$ and $B$ are $0.79 \mathrm{~cm}(D)$ Corresponding ex vivo fluorescent images from left to right of kidney, liver, MIN6, pancreas, spleen and muscle tissues excised at 30 min and (E) 4 h post-i.v. injection of E4x12-Cy7. Scale bars in D and E are $0.4 \mathrm{~cm}$. (F) Quantification of the ex vivo organs by drawing regions of interest (ROIs) around the tissue outlines using the white field images. 


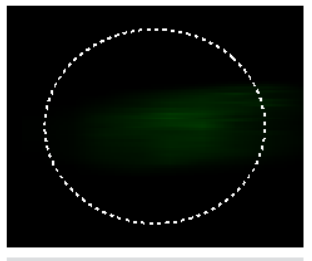

saline

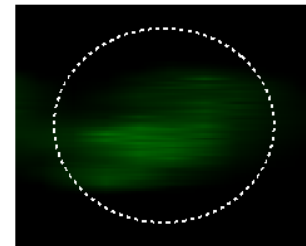

blocking

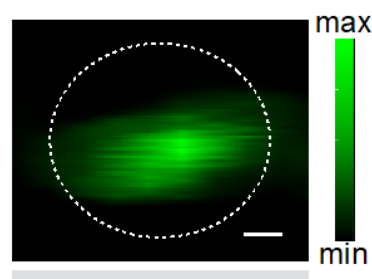

E4x12-Cy7

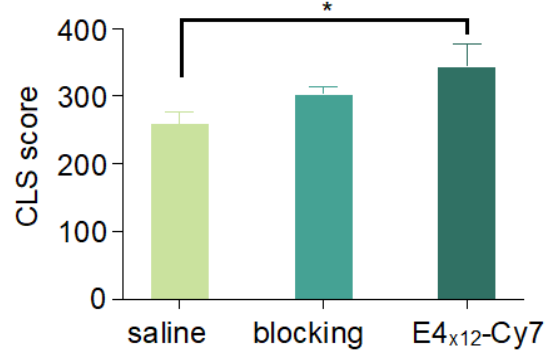

Figure S3. The ex vivo optoacoustic signal of kidneys between non injected, blocking and injected. From left to right are the MSOT images of the kidney from a mouse that was injected with saline, exendin-4 $(151 \mu \mathrm{g})$ followed by the injection of $\mathrm{E}_{4 \times 12-\mathrm{Cy}} \mathrm{7}(57 \mu \mathrm{g}, 20$ mins time interval (blocking), and $57 \mu \mathrm{g}$ of $\mathrm{E} 4 \times 12-\mathrm{Cy} 7$ (left) and the corresponding quantification (right). There is a statistically significant difference between mice that were injected with saline and $\mathrm{E} 4 \times 12-\mathrm{Cy} 7\left(\mathrm{p}^{*}=0.0178\right)$. Scale bar is $2.5 \mathrm{~mm}$. 

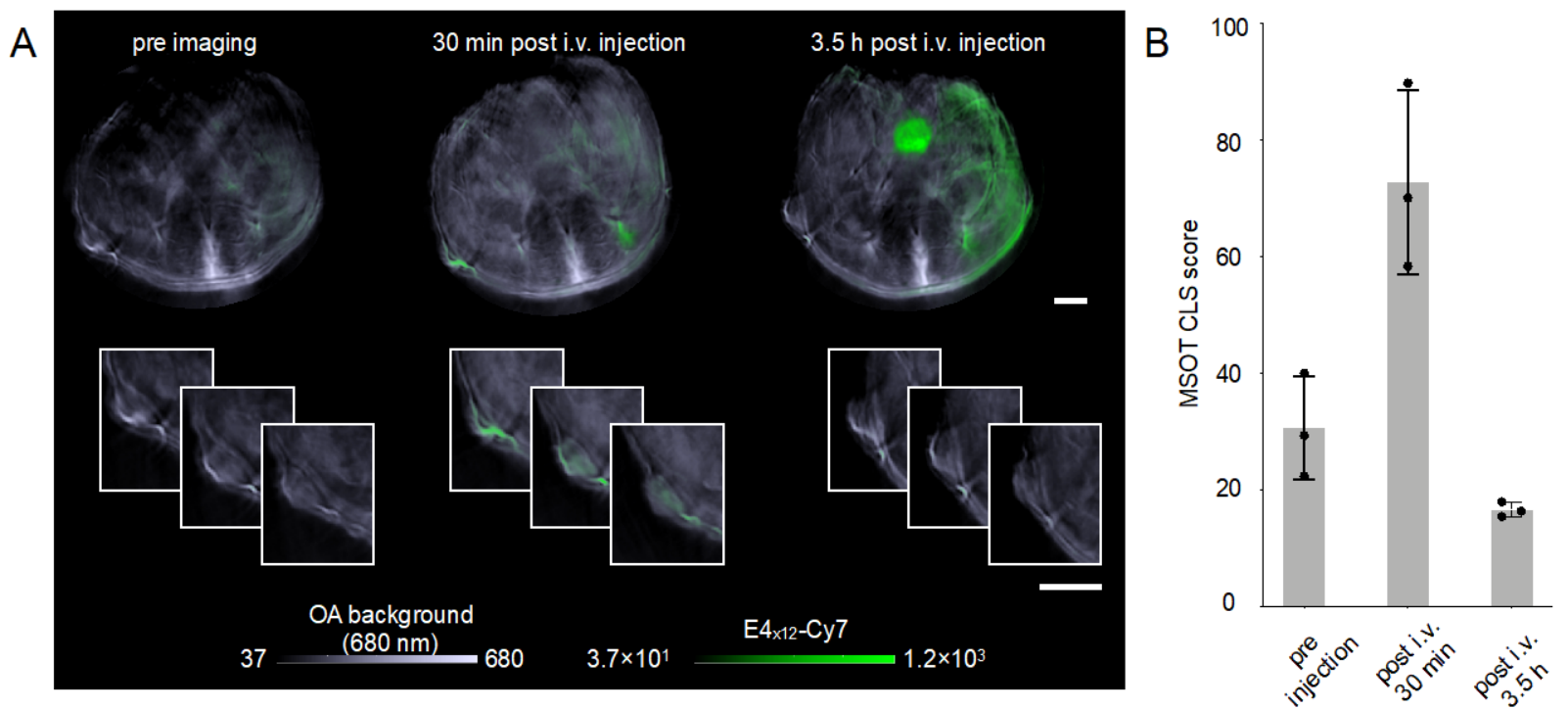

Figure S4. In vivo multi-spectral optoacoustic evaluation of $\mathrm{E} 4 \times 12-\mathrm{Cy} 7$ at different timepoints. (A) Optoacoustic image reconstruction showing transverse 2D projection of mice at the region of interest before and $30 \mathrm{~min}$ after intravenous injection of E4x12-Cy7 $(6.7 \mathrm{mg} / \mathrm{kg}$ ) before (left), $30 \mathrm{~min}$ post-i.v. injection (middle) and $3.5 \mathrm{~h}$ post-i.v. injection (right). Overall optoacoustic signal at $680 \mathrm{~nm}$ (greyscale) is overlaid on top of multispectrally unmixed signals showing, E4×12-Cy7 channel (green channel). Both scale bars are $2.5 \mathrm{~mm}$. (B) Optoacoustic signal quantification after multi-spectral unmixing. 